\title{
On InSAR AMBIGUITY RESOLUTION FOR DEFORMATION MONITORING
}

\author{
P.J.G. Teunissen \\ Department of Earth Observation and Space Systems (DEOS) \\ Delft University of Technology \\ Kluyverweg 1, Delft, The Netherlands \\ Fax: ++ 3115278 3711; E-mail: P.J.G.Teunissen@lr.tudelft.nl
}

\begin{abstract}
Integer carrier phase ambiguity resolution is the key to fast and highprecision satellite positioning and navigation. It applies to a great variety of current and future models of GPS, modernized GPS and Galileo. It also applies to stacked radar interferometry for deformation monitoring, see e.g. [Hanssen, et al, 2001]. In this contribution we apply the integer least-squares' principle to the rank defect model of stacked InSAR carrier phase data. We discuss two ways of dealing with the rank defect for ambiguity resolution. One is based on the use of a priori data, the other is based on the use of an interval constraint on the deformation rate.
\end{abstract}

\section{INTEGER LEAST-SQUARES (ILS)}

Consider the system of observation equations

$$
y=A x+B z+e
$$

where $y \in R^{m}$ is the vector of observations, $x \in R^{p}$ and $z \in Z^{n}$ are the vectors of unknown parameters and $e \in R^{m}$ is the noise vector. Matrix $(A, B)$ is given and assumed to be of full column rank. The structure of the system (1) is typical for carrier phase GPS applications. In that case $y$ consists of the carrier phase and pseudo range data, $z$ consists of the integer double difference ambiguities and $x$ consists of the baseline components and possibly other real-valued parameters (e.g. atmospheric delay parameters). The model is however also applicable to radar interferometric phase ambiguity resolution problems, see e.g. [Bamler and Hartl, 1998], [Hanssen, 2001], [Hanssen et al, 2001], [Kampes and Hanssen, 2004], [Kampes, 2005].

In order to solve (1) in a least-squares sense, we follow the approach of [Teunissen, 1993]. Let $Q_{y}$ be the variance matrix of $y$ and let $\|.\|_{Q_{y}^{-1}}^{2}=(.)^{T} Q_{y}^{-1}($.$) . We first decompose$ $\|e\|_{Q_{y}^{-1}}^{2}$ into a sum of three quadratic terms:

$$
\|e\|_{Q_{y}^{-1}}^{2}=\|y-A x-B z\|_{Q_{y}^{-1}}^{2}=\|\hat{e}\|_{Q_{y}^{-1}}^{2}+\left.\|\hat{z}-z\|\right|_{Q_{z}^{-1}} ^{2}+\|\hat{x}(z)-x\|_{Q_{\hat{x} \mid z}^{-1}}^{2}
$$


in which $\hat{e}=y-A \hat{x}-B \hat{z}, \hat{x}$ and $\hat{z}$ are the least-squares solutions for $x$ and $z$, respectively, assuming that $z$ is real-valued instead of integer-valued, and

$$
\hat{x}(z)=\left(A^{T} Q_{y}^{-1} A\right)^{-1} A^{T} Q_{y}^{-1}(y-B z)
$$

is the least-squares solution of $x$ based on the assumption that $z$ is known. In GPS terminology, the solutions $\hat{x}$ and $\hat{z}$ are also referred to as the 'float' solutions. Solution $\hat{x}(z)$ is a conditional least-squares solution. Matrices $Q_{\hat{z}}$ and $Q_{\hat{x} \mid z}$ are the variance matrices of $\hat{z}$ and $\hat{x}(z)$, respectively. Note that the 'float' solutions $\hat{x}$ and $\hat{z}$ would not be unique in case matrix $(A, B)$ is rank defect.

From (2) follows that

$$
\min _{x \in R^{p}, z \in Z^{n}}\|y-A x+B z\|_{Q_{y}^{-1}}^{2}=\|\hat{e}\|_{Q_{y}^{-1}}^{2}+\min _{z \in Z^{n}}\left\{\|\hat{z}-z\|_{Q_{\hat{z}}^{-1}}^{2}+\min _{x \in R^{p}}\|\hat{x}(z)-x\|_{Q_{\hat{x} \mid z}^{-1}}^{2}\right\}
$$

This shows that the least-squares solutions for $x \in R^{p}$ and $z \in Z^{n}$ are given as

$$
\check{z}=\arg \min _{z \in Z^{n}}\|\hat{z}-z\|_{Q_{z}^{-1}}^{2} \text { and } \check{x}=\hat{x}(\check{z})
$$

In [Teunissen, 1995] it is shown how $\check{z}$ can be computed in an efficient manner by means of the LAMBDA method. In [Teunissen, 1999] it is shown that the integer least-squares estimator $\check{z}$ is optimal in the sense that it maximizes the probability of correct integer estimation. For a brief review of statistical carrier phase ambiguity resolution, see e.g. [Teunissen, 2001].

The probability of correct integer estimation (the so-called success-rate) is driven by the variance matrix of $\hat{z}$. The ADOP (Ambiguity Dilution of Precision), defined as

$$
\mathrm{ADOP}=\left|Q_{\hat{z}}\right|^{\frac{1}{2 n}} \quad(\text { cycle })
$$

is a simple scalar measure for the precision of $\hat{z}$. It is the geometric mean of the conditional standard deviations of $\hat{z}$. It has the advantage of being invariant for the class of admissible integer transformations. The ADOP can be used to get a first quick impression on the model's ability to achieve successful ambiguity resolution. Fo this to be the case, the ADOP needs to be small enough (e.g. well below the cycle level).

\section{InSAR ILS WITH DEFORMATION DATA}

One of the simplest models of phase ambiguity resolution for stacked radar interferometry takes the form (see e.g. [Hanssen et al., 2001]),

$$
y_{i}=a_{i} x+z_{i}+e_{i}, i=1, \ldots, n
$$

with $y_{i}$ the observed phase difference between two permanent scatterers (expressed in cycles), $x$ the unknown deformation rate, $z_{i}$ the unknown integer ambiguity, $e_{i}$ the noise term, and $a_{i}=\frac{2}{\lambda} \Delta t_{i}$, where $\lambda$ denotes the wavelength (e.g. $\left.5.8 \mathrm{~cm}\right)$ and $\Delta t_{i}$ is a time interval (expressed in years) between the current time and a reference time. The model can be written in vector form as

$$
y=a x+z+e
$$


This model appears to be a special case of (1), since $A \mapsto a, B \mapsto I, p=1$ and $m=n$. Note however that the condition of having a coefficient matrix of full column rank is not met anymore, since matrix $\left(a, I_{n}\right)$ has a rank defect of one. Hence, the system contains insufficient information to compute a unique 'float' solution for $x$ and $z$. This situation can be remedied by including data $x_{o}$ on the deformation rate $x$. This could be actual data (from earlier studies) or pseudo-data. As a result we obtain the full rank model

$$
\left[\begin{array}{c}
y \\
x_{0}
\end{array}\right]=\left[\begin{array}{c}
a \\
1
\end{array}\right] x+\left[\begin{array}{c}
I \\
0
\end{array}\right] z+\left[\begin{array}{c}
e \\
e_{x}
\end{array}\right] \text { with variance matrix }\left[\begin{array}{cc}
Q_{y} & 0 \\
0 & \sigma_{x}^{2}
\end{array}\right]
$$

in which $\sigma_{x}^{2}$ is used to express the a priori uncertainty of the deformation rate. Now that the model is of full rank, the method of the previous section can be applied again. This is the approach which has been followed in [Hanssen et al, 2001].

To determine the ADOP, we need the variance matrix of $\hat{z}$. It is given as $Q_{\hat{z}}=$ $Q_{y}+\sigma_{x}^{2} a a^{T}$, from which the ADOP follows as

$$
\mathrm{ADOP}=\left|Q_{\hat{z}}\right|^{\frac{1}{2 n}}=\left|Q_{y}\right|^{\frac{1}{2 n}}\left(1+\sigma_{x}^{2} a^{T} Q_{y}^{-1} a\right)^{\frac{1}{2 n}}
$$

With $Q_{y}=\sigma_{\phi}^{2} I_{n}, a_{i}=\frac{2}{\lambda} \Delta t_{i}$ and small $\sigma_{\phi}$, we get

$$
\mathrm{ADOP} \approx \sigma_{\phi}\left(\frac{2}{\lambda} \frac{\sigma_{x}}{\sigma_{\phi}} \sqrt{\sum \Delta t_{i}^{2}}\right)^{\frac{1}{n}} \quad(\text { cycle })
$$

This simple result can be used to get a quick impression of whether the model has enough strength for successful ambiguity resolution.

\section{InSAR ILS WITH DEFORMATION CONSTRAINT}

Instead of working with a priori data on the deformation rate $x$, one may also consider using a constraint in the form of an interval $\alpha \leq x \leq \beta$, with $\alpha$ and $\beta$ given. In practice one usually has a fair idea over which range one can expect the deformation to occur. In that case it is not difficult to choose $\alpha$ and $\beta$. Hence, the ILS-problem now becomes

$$
\min _{x \in R, z \in Z^{n}}\|y-a x-z\|_{Q_{y}^{-1}}^{2} \text { subject to } \alpha \leq x \leq \beta
$$

Note that we could have added the constraint also to the model based on the a priori deformation data. In the following, however, we will work with the model (12). The procedure for solving it can also be used for the model based on the a priori deformation data.

Unconstrained minimization Let us first consider minimizing the least-squares' objective function without the constraint. With the use of the orthogonal projectors $P_{a}=$ $a\left(a^{T} Q_{y}^{-1} a\right)^{-1} a^{T} Q_{y}^{-1}$ and $P_{a}^{\perp}=I_{n}-P_{a}$, we have

$$
\begin{aligned}
\min _{x \in R, z \in Z^{n}}\|y-a x-z\|_{Q_{y}^{-1}}^{2} & =\min _{x \in R, z \in Z^{n}}\left\{\left\|P_{a}^{\perp}(y-z)\right\|_{Q_{y}^{-1}}^{2}+\left\|P_{a}(y-z)-a x\right\|_{Q_{y}^{-1}}^{2}\right\} \\
& =\min _{z \in Z^{n}}\left\{\left\|P_{a}^{\perp}(y-z)\right\|_{Q_{y}^{-1}}^{2}+\min _{x \in R}\left\|P_{a}(y-z)-a x\right\|_{Q_{y}^{-1}}^{2}\right\} \\
& =\min _{z \in Z^{n}}\left\{\left\|P_{a}^{\perp}(y-z)\right\|_{Q_{y}^{-1}}^{2}+\min _{x \in R}\|\hat{x}(z)-x\|_{a^{T} Q_{y}^{-1} a}^{2}\right\}
\end{aligned}
$$


with $\hat{x}(z)=\left(a^{T} Q_{y}^{-1} a\right)^{-1} a^{T} Q_{y}^{-1}(y-z)$. This shows that the solution is given as

$$
\check{z}=\arg \min _{z \in Z^{n}}\left\|P_{a}^{\perp}(y-z)\right\|_{Q_{y}^{-1}}^{2} \text { and } \check{x}=\hat{x}(\check{z})
$$

This result seems to imply that in order to find $\check{z}$, we need to search over the complete space of integers $Z^{n}$. Clearly, this is not practically feasible. Fortunately, we can confine the search to a subset of $Z^{n}$.

The relevant integer vectors Let $S: R^{n} \mapsto Z^{n}$ be the integer map of componentwise integer rounding. Then all $y \in R^{n}$ which are mapped by $S$ to $z \in Z^{n}$ form the set

$$
S_{z}=\left\{y \in R^{n} \mid S(y)=z\right\}
$$

This set is the $n$-dimensional 'unit-cube' centred at $z$. Note, since $Q_{y}$ is assumed to be a diagonal matrix, that $S$ has the property $\min _{z \in Z^{n}}\|y-z\|_{Q_{y}^{-1}}^{2}=\|y-S(y)\|_{Q_{y}^{-1}}^{2}$. We may therefore decompose the least-squares' objective function also as

$\min _{x \in R, z \in Z^{n}}\|y-a x-z\|_{Q_{y}^{-1}}^{2}=\min _{x \in R}\left\{\min _{z \in Z^{n}}\|y-a x-z\|_{Q_{y}^{-1}}^{2}\right\}=\min _{x \in R}\|y-a x-S(y-a x)\|_{Q_{y}^{-1}}^{2}$

This shows that the relevant integer vectors are those which are the centres of the 'unitcubes' through which the line $y-a x$ passes. They form the set

$$
\Omega_{z}=\left\{z \in Z^{n} \mid S(y-a x)=z, x \in R\right\}
$$

Thus instead of (14) we may now write

$$
\check{z}=\arg \min _{z \in \Omega_{z}}\left\|P_{a}^{\perp}(y-z)\right\|_{Q_{y}^{-1}}^{2} \text { and } \check{x}=\hat{x}(\check{z})
$$

However, since the range of $x$ has not yet been bounded, the set $\Omega_{z}$ still contains an infinite number of integer vectors.

The finite set $\Omega_{z}^{\prime}$ We will now make use of the constraint $\alpha \leq x \leq \beta$. This allows us to reduce the infinite set $\Omega_{z}$ to the finite set

$$
\Omega_{z}^{\prime}=\left\{z \in Z^{n} \mid S(y-a x)=z, \alpha \leq x \leq \beta\right\}
$$

Hence, we are now only considering a line segment of length $\sqrt{a^{T} a}(\beta-\alpha)$ of the line $y-a x$. Note that the length of the line segment is an approximate upper bound for the number of integer vectors contained in $\Omega_{z}^{\prime}$.

Finding the integer vectors $z \in \Omega_{z}^{\prime}$ To find all integer vectors that satisfy $S(y-a x)=z$ for $\alpha \leq x \leq \beta$, we first make the problem a bit more tractable. Let $x^{\prime}=x-\alpha$, $z^{\prime \prime}=S(y-a \alpha), z^{\prime}=z-z^{\prime \prime}$, and $y^{\prime}=y-a \alpha-z^{\prime \prime}$. Then $z=S(y-a x)=S\left(y^{\prime}-a x^{\prime}\right)+z^{\prime \prime}$ and thus

$$
z^{\prime}=S\left(y^{\prime}-a x^{\prime}\right) \text { with } 0 \leq x^{\prime} \leq \gamma=(\beta-\alpha) \text { and } y^{\prime} \in S_{0}
$$

Hence, $x^{\prime}$ is nonnegative and all components of $y^{\prime}$ lie within the interval $\left[-\frac{1}{2},+\frac{1}{2}\right]$. Note that $z^{\prime}=S\left(y^{\prime}-a x^{\prime}\right)$ is equivalent to the $n$ intervals $z_{i}^{\prime}-\frac{1}{2} \leq y_{i}^{\prime}-a_{i} x^{\prime} \leq z_{i}^{\prime}+\frac{1}{2}, i=1, \ldots, n$. 
To find the elements of $\Omega_{z}^{\prime}$, one can now proceed as follows. Consider for each $i$ the graph of the line $\epsilon_{i}(x)=y_{i}^{\prime}-a_{i} x^{\prime}$ as function of $x^{\prime}$. The line starts at the point $\left(0, y_{i}^{\prime}\right)$, where for each $i, y_{i}^{\prime} \in\left[-\frac{1}{2},+\frac{1}{2}\right]$. The line may be located in either the first or the fourth quadrant, depending on whether $a_{i} \leq 0$ or $a_{i} \geq 0$. Starting with $x^{\prime}=0$, the first candidate integer vector is $z^{\prime}=0$. The next candidate integer vector follows from which of the $n$ lines $\epsilon_{i}(x)$ first crosses one of the levels $\pm \frac{1}{2}$ modulus 1 . For instance, if it is the line $\epsilon_{j}(x)$ which first crosses the level $\pm \frac{1}{2}$, then the second candidate integer vector is given as $z^{\prime}=(\ldots, 0,1,0, \ldots)^{T}$, with the 1 in slot $j$. In this way one can proceed collecting all candidate integer vectors $z^{\prime}$ for the range $0 \leq x^{\prime} \leq \gamma$. From them the elements of $\Omega_{z}^{\prime}$ are obtained as $z=z^{\prime}+S(y-a \alpha)$.

Computing the final solution Now that the finite set $\Omega_{z}^{\prime}$ is given, we compute

$$
\check{z}=\arg \min _{z \in \Omega_{z}^{\prime}}\left\|P_{a}^{\perp}(y-z)\right\|_{Q_{y}^{-1}}^{2} \text { and } \check{x}=\hat{x}(\check{z})
$$

On this result one last check has to be performed, namely whether the constraint $\alpha \leq$ $\check{x}=\hat{x}(\check{z}) \leq \beta$ is satisfied or not. If it is, then (21) is the solution sought. If it is not, then the solution is given by one of the two endpoints of the line segment, $x=\alpha$ or $x=\beta$. The solution is $x=\alpha$ if $\|y-a \alpha-S(y-a \alpha)\|_{Q_{y}^{-1}}^{2}<\|y-a \beta-S(y-a \beta)\|_{Q_{y}^{-1}}^{2}$. Note that in practice one may question the choice of the constraint if one of the two endpoints turns out to be the solution. The purpose of the constraint in our present application is namely to be able to construct a finite integer search space $\Omega_{z}^{\prime}$, but it is not chosen so as to influence the final solution. Thus if one of the two endpoints turns out to be the solution, it is likely that the constraint has been chosen too strict.

\section{A POSTERIORI PROBABILITY OF THE INTEGER SOLUTION}

Once the integer vector $\check{z}$ has been computed, one would like to know whether one can have any trust in the solution. Intuitively, this boils down to a comparison of the values taken by $\left\|P_{a}^{\perp}(y-z)\right\|_{Q_{y}^{-1}}^{2}$ for $z \in \Omega_{z}^{\prime}$. One would then have more trust in the solution if $\left\|P_{a}^{\perp}(y-\check{z})\right\|_{Q_{y}^{-1}}^{2}$ is significantly the smallest value, than when $\left\|P_{a}^{\perp}(y-\check{z})\right\|_{Q_{y}^{-1}}^{2}$ is comparable to one or more of the other values. As shown below this is what is measured by the conditional distribution of $z$.

The following assumptions are made: $y \mid x, z \sim N\left(a x+z, Q_{y}\right)$, with conditional probability density function (pdf) $f(y \mid x, z)$. Thus $f(y \mid x, z) \propto \exp \left\{-\frac{1}{2}|| y-a x-z \|_{Q_{y}^{-1}}^{2}\right\}$ (where $\propto$ stands for proportional to). $x$ is uniformly distributed over the interval $[\alpha, \beta]$. Its pdf $g(x)$ is therefore proportional to the indicator function $\delta_{\alpha, \beta}(x)$ of the interval, $g(x) \propto \delta_{\alpha, \beta}(x)$. For the distribution of $z$, we assume a noninformative prior. $x$ and $z$ are assumed to be independent and the distribution of $z$ is denoted as $h(z)$. We therefore have for the joint distribution $f(x, y, z)=f(y \mid x, z) g(x) h(z)$, from which the a posteriori distribution of $z$ follows as

$$
h(z \mid y)=\frac{\int f(y \mid x, z) g(x) d x}{\sum_{z \in Z^{n}} \int f(y \mid x, z) g(x) d x}=\frac{\exp \left\{-\frac{1}{2}\left\|P_{a}^{\perp}(y-z)\right\|_{Q_{y}^{-1}}^{2}\right\} k(z)}{\sum_{z \in Z^{n}} \exp \left\{-\frac{1}{2}|| P_{a}^{\perp}(y-z) \|_{Q_{y}^{-1}}^{2}\right\} k(z)}
$$


where

$$
k(z)=\frac{1}{\sigma \sqrt{2 \pi}} \int_{\alpha}^{\beta} \exp \left\{-\frac{1}{2}\left(\frac{x-\hat{x}(z)}{\sigma}\right)^{2}\right\} d x \text { with } \sigma^{2}=\frac{1}{a^{T} Q_{y}^{-1} a}
$$

For sufficiently small $\sigma, k(z)$ will be close to one for $\hat{x}(z) \in[\alpha, \beta]$ and close to zero outside this range. Under this assumption (22) may be approximated as

$$
h(z \mid y) \approx \frac{\exp \left\{-\frac{1}{2}\left\|P_{a}^{\perp}(y-z)\right\|_{Q_{y}^{-1}}^{2}\right\}}{\sum_{z \in \Omega_{z}^{\prime}} \exp \left\{-\frac{1}{2} \|\left. P_{a}^{\perp}(y-z)\right|_{Q_{y}^{-1}} ^{2}\right\}}
$$

Note that $\check{z}$ is the maximizer of the right hand side. The a posteriori probability of the integer solution (i.e. the probability conditioned on the data) is given as $h(\check{z} \mid y)$. The closer this value is to one, the more trust one will have in the computed solution.

\section{REFERENCES}

Bamler, R. and Hartl, P. (1998): Synthetic aperture radar interferometry. Inverse Problems, 14, R1-R54.

Hanssen, R.F. (2001): Radar Interferometry: Data Interpretation and Error Analysis. Kluwer Academic Publishers, Dordrecht.

Hanssen, R.F., P.J.G. Teunissen and P. Joosten (2001): Phase ambiguity resolution for stacked radar interferometric data. In: Proc. KIS2001, International Symposium on Kinematic Systems in Geodesy, Geomatics and Navigation, Banff, Canada, pp. 317-320.

Kampes, B.M. and R.F. Hanssen (2004): Ambiguity resolution for permanent scatterer interferometry. IEEE Transactions on Geoscience and Remote Sensing, 42(11), 2446-2453.

Kampes, B.M. (2005): Displacement Parameter Estimation using Permanent Scatterer Interferometry. Netherlands Geodetic Commission. Publications on Geodesy, 168 p.

Teunissen, P.J.G. (1993): Least-squares estimation of the integer GPS ambiguities. Invited Lecture, Section IV Theory and Methodology, IAG General Meeting, Beijing, China, August 1993. Also in: LGR Series, No. 6, Delft Geodetic Computing Centre.

Teunissen, P.J.G. (1995): The least-squares ambiguity decorrelation adjustment: a method for fast GPS integer ambiguity estimation. Journal of Geodesy, 70: 65-82.

Teunissen, P.J.G. (1999): An optimality property of the integer least- squares estimator. Journal of Geodesy, 73: 587-593.

Teunissen, P.J.G. (2001): Statistical GNSS carrier phase ambiguity resolution: a review. IEEE, 0-7803-7011-2/01, pp. 4-12.

Received: 2006-01-23,

Reviewed: 2006-02-03, by W. Pachelski,

Accepted: 2006-02-27. 